\title{
Electronic Integrated Disease Surveillance System and Pathogen Asset Control System
}

\author{
Authors: \\ Tom G. Wahl ${ }^{1}$ \\ Aleksey V. Burdakov ${ }^{1}$ \\ Andrey O. Oukharov \\ Azamat K. Zhilokov ${ }^{1}$ \\ Affiliations: \\ ${ }^{1}$ Black and Veatch, MosAlarko \\ Plaza One, Moscow, Russia \\ Correspondence to: \\ Tom Wahl \\ Email: \\ wahlt@bv.com \\ Postal address: \\ Black and Veatch, MosAlarko \\ Plaza One, 16 Marksistskaya \\ St., Moscow 109147, Russia \\ How to cite this proceeding: \\ Wahl, T.G., Burdakov, A.V., \\ Oukharov, A.O. \& Zhilokov, \\ A.K., 2012, 'Electronic \\ Integrated Disease \\ Surveillance System and \\ Pathogen Asset Control \\ System', Onderstepoort \\ Journal of Veterinary \\ Research 79(2), Art. \#455, \\ 5 pages. http://dx.doi. \\ org/10.4102/ojvr.v79i2.455 \\ Note: \\ Proceedings of the \\ Conference of the Southern \\ African Centre for Infectious \\ Disease Surveillance 'One \\ Health' held at the National \\ Institute for Communicable \\ Diseases, Johannesburg, July \\ 2011.
}

C 2012. The Authors. Licensee: AOSIS OpenJournals. This work is licensed under the Creative Commons Attribution License.
Electronic Integrated Disease Surveillance System (EIDSS) has been used to strengthen and support monitoring and prevention of dangerous diseases within One Health concept by integrating veterinary and human surveillance, passive and active approaches, case-based records including disease-specific clinical data based on standardised case definitions and aggregated data, laboratory data including sample tracking linked to each case and event with test results and epidemiological investigations. Information was collected and shared in secure way by different means: through the distributed nodes which are continuously synchronised amongst each other, through the web service, through the handheld devices. Electronic Integrated Disease Surveillance System provided near real time information flow that has been then disseminated to the appropriate organisations in a timely manner. It has been used for comprehensive analysis and visualisation capabilities including real time mapping of case events as these unfold enhancing decision making. Electronic Integrated Disease Surveillance System facilitated countries to comply with the IHR 2005 requirements through a data transfer module reporting diseases electronically to the World Health Organisation (WHO) data center as well as establish authorised data exchange with other electronic system using Open Architecture approach.

Pathogen Asset Control System (PACS) has been used for accounting, management and control of biological agent stocks. Information on samples and strains of any kind throughout their entire lifecycle has been tracked in a comprehensive and flexible solution PACS.

Both systems have been used in a combination and individually. Electronic Integrated Disease Surveillance System and PACS are currently deployed in the Republics of Kazakhstan, Georgia and Azerbaijan as a part of the Cooperative Biological Engagement Program (CBEP) sponsored by the US Defense Threat Reduction Agency (DTRA).

\section{Problem statement}

Infectious diseases in the twenty-first century continue to cause economic and social disruptions becoming more severe due to the emerging diseases often of a zoonotic nature, active travel increasing potential of regional and international epidemics and pandemics, and potential bioterrorist threats.

To adequately address these growing threats countries have to strengthen and improve their capability to perform early detection and rapid reporting of infectious disease situation and outbreaks, to timely and accurately verify presence or absence of high-consequence pathogens, and to comprehensively and rapidly respond to care for infected patients and reduce exposure of the wider population, and the accidental and/or deliberate release of high-consequence pathogens.

There have been a number of attempts to improve detection, reporting and respond capabilities with the modern information and communications technology. However, most of the advances in this field tend to either concentrate on isolated vertical segments (e.g. HIV, TB or Malaria), separately address human or veterinary areas, and be isolated from diagnostics (laboratory) and clinical data sources, be disintegrated with the international information systems (World Health Organisation [WHO] and World Organisation for Animal Health [OIE]), or be tailored only for certain environment and disease surveillance priorities without an ability to adjust.

Thus there is a need for a comprehensive information and communications technology solution, which would overcome the challenges of existing solutions and facilitate in early detection, rapid reporting and response. The creation of this comprehensive solution would require significant effort from the epidemiology and diagnostics experts society as well as investment in development and implementation of the solution. 


\section{Methods and approach}

For the 10 years the United States Defense Threat Reduction Agency (DTRA) has been actively implementing the Cooperative Biological Engagement Program (CBEP), which goals closely match infectious disease threats most of the countries face:

- combat bioterrorism and prevent the proliferation of biological weapons-related technology, pathogens and expertise

- enhance host governments' disease surveillance systems to detect and report bioterrorism attacks, epidemics and potential pandemics.

To address these goals two information and communications technology solutions were created, (1) Electronic Integrated Disease Surveillance System (EIDSS) and (2) Pathogen Asset Control System (PACS). Electronic Integrated Disease Surveillance System facilitates in early detection, rapid reporting and response to disease outbreaks, whilst PACS improves security of dangerous pathogens stored in the laboratories.

\section{Method 1: Electronic surveillance using Electronic Integrated Disease Surveillance System}

Electronic Integrated Disease Surveillance System is an electronic system intended to facilitate in collecting, notifying, sharing and analysing surveillance data. It consists of several modules listed below together with a specification of tracked data:

- Human module

- demographic data

- disease-specific clinical data based on standard case definitions

- epidemiological investigations

- sample and laboratory tests tracking linked to a specific case

- aggregate cases.

- Veterinary module

- avian and livestock cases

- farm or owner information

- disease-specific clinical data based on standard case definitions

- epidemiological investigations

- sample and laboratory tests tracking linked to a specific case

- penside tests

- aggregate cases

- active surveillance data.

- Laboratory

- samples, tests assignment, tests results and batch tests

- aliquots and derivatives

- transfer in or out operations

- links to clinical case data.

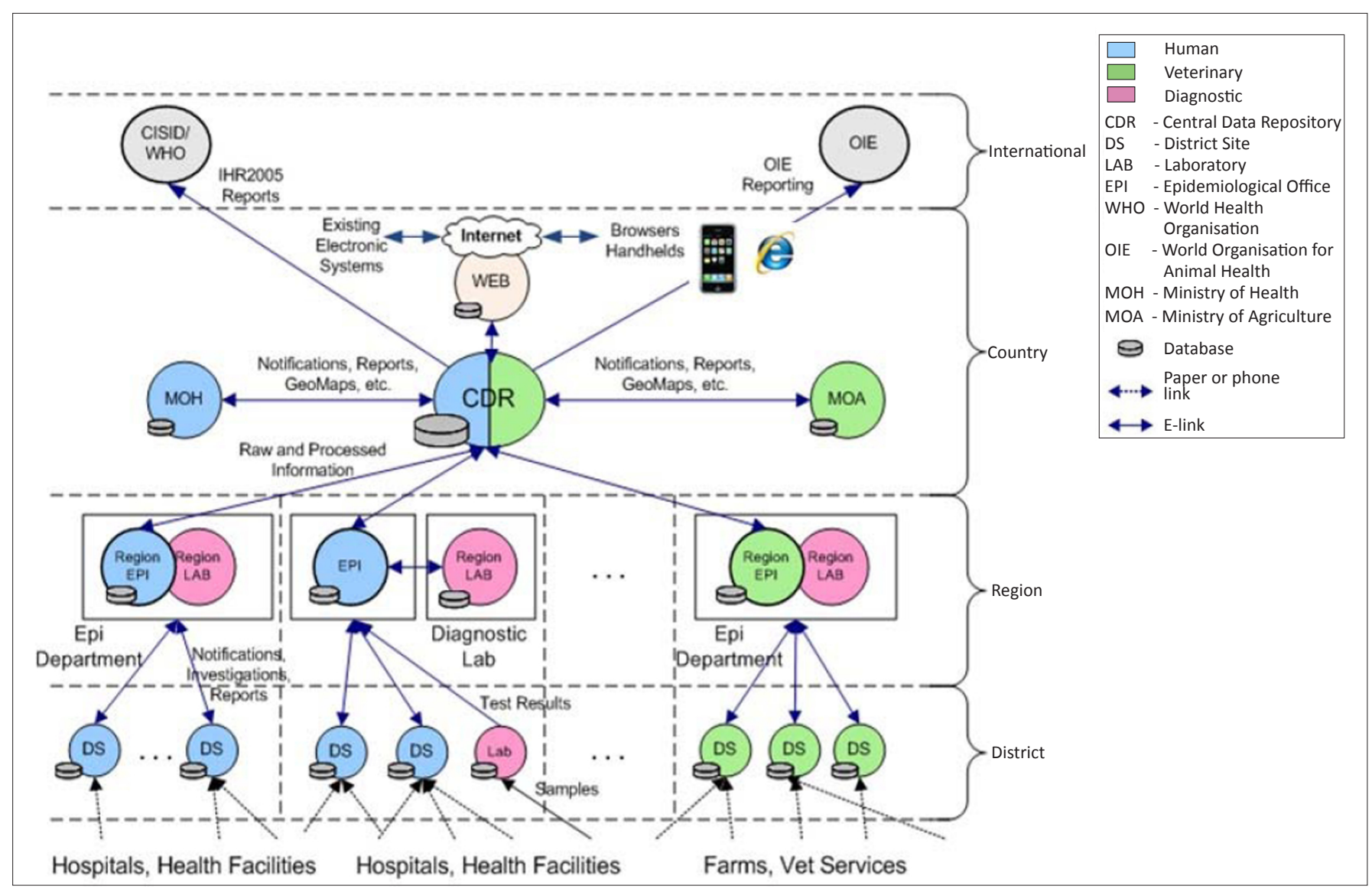

FIGURE 1: Typical Electronic Integrated Disease Surveillance System architecture. 
- Analysis

- customised and predefined reports

- statistic analysis

- different ways of data representation: grids, charts, maps.

Information can be collected and shared through these modules in several ways providing a near real-time information flow that can be then disseminated to the appropriate organisations in a timely manner. Figure 1 represents typical EIDSS hierarchy covering different administrative levels: district, region, country.

The first way to collect information is through a number of distributed nodes which are continuously synchronised amongst each other. Let us say a veterinary case is registered in a district. Once this information is entered to the system, replication starts and transfers the case to the region level site, which in turn send it to the Central Data Repository (CDR), then it goes other sites which are supposed to get such information. Bi-directional regular data exchange amongst sites guaranties that all participants get actual and up-todate information. If a node gets off-line it keeps working in disconnected mode and will be synchronised with other nodes once it gets on-line.

The EIDSS Web server is the other way to get information into the system (Figure 2). Data entered through the web site is distributed to other nodes of the system and available for review and editing according to users' permissions.

Access to EIDSS from handheld devices is the third entry point (Figure 2). Web portal designed for handheld devices provides simplified interface and support a variety of devices. Any combination of these methods can be used depending on infrastructure, organisational and other issues. In a combination with using of commercial 'off-the-shelf' readily available generic hardware it makes EIDSS suitable for environments with different challenges.

\section{Methods and features}

Following One Health approach EIDSS integrates human and veterinary surveillance data along with a laboratory information. For example, it is possible to create an outbreak record in the system which ties together several human and veterinary cases. One can also link several veterinary cases together. Adding a laboratory piece gives the opportunity diagnose a case with a most recent laboratory data. For instance, case entered in the veterinary service is transferred to the laboratory and available through the EIDSS lab module. As soon as test results are registered for this case in the laboratory they will be transferred to the veterinary service site and can be used for further investigations.

Flexibility and scalability is one of the fundamental principles of the EIDSS. One example of such flexibility is that cases can be tracked with different levels of specification: case-based tracking with a specific case definitions and investigation information, when every case is registered as individual record, can be used for especially dangerous diseases, case-based tracking with just an emergency notification information can be used for other important diseases, aggregate reporting which is supposed to track just summary data from district or regions for a certain period of time. Electronic Integrated Disease Surveillance System also allows to switch between different approaches depending on the current situation. Case definitions used in the system also can be adjusted according to the specific requirements.

Electronic Integrated Disease Surveillance System supports different types of surveillance: passive surveillance (casebased and aggregate) is available for human and veterinary diseases, active surveillance is supported for veterinary disease, vector surveillance is planned to be released in the next version.

Comprehensive analysis modules give users various capabilities to investigate and present collected data. Using either predefined reports which duplicates officiallyapproved paper forms or customisable reports where one can build a report as needed, users are able to get access to any variables in the database and explore human, veterinary and lab. The GIS component of the system allows attaching geo-coordinates to cases and map data in addition to charts and grid representations (Figure 2).

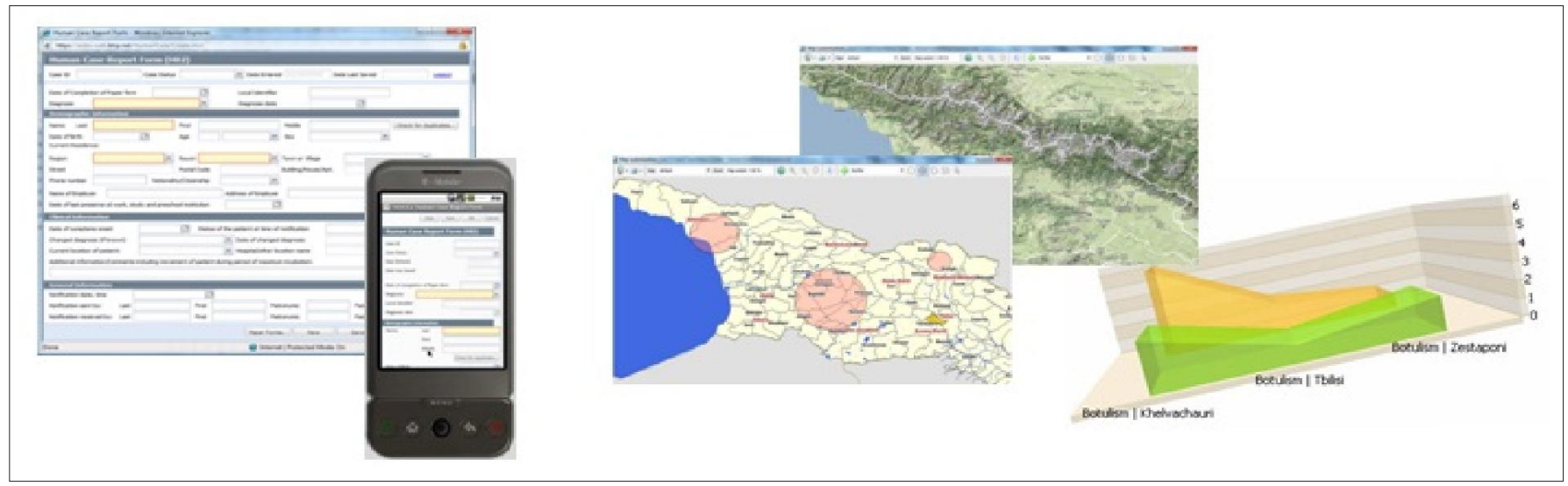

FIGURE 2: Web and/or handheld access to Electronic Integrated Disease Surveillance System: data analysis. 


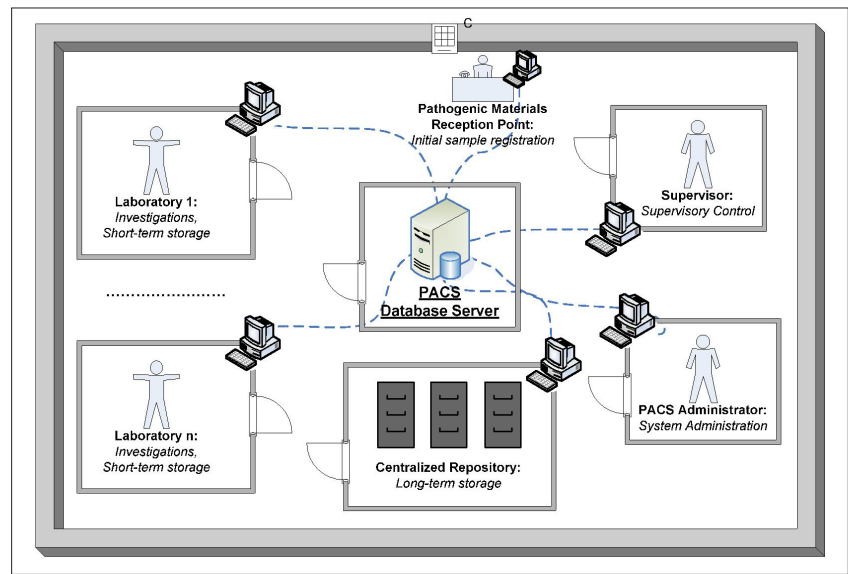

FIGURE 3: Pathogen Asset Control System conceptual schema.

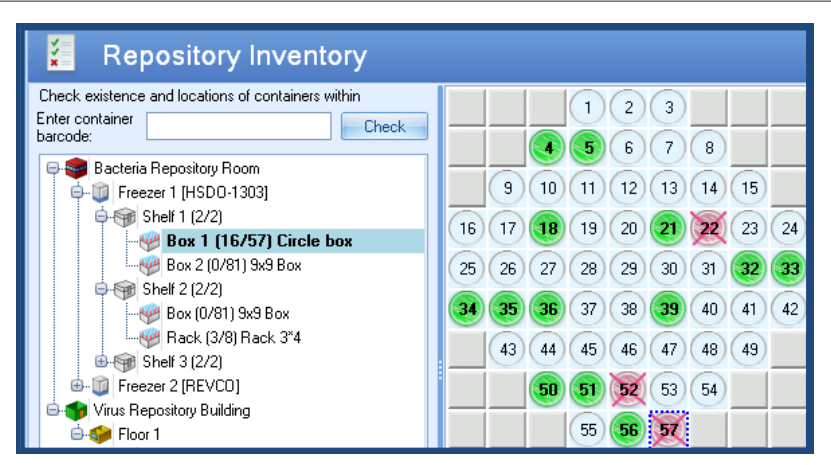

FIGURE 4: Repository inventory audit.

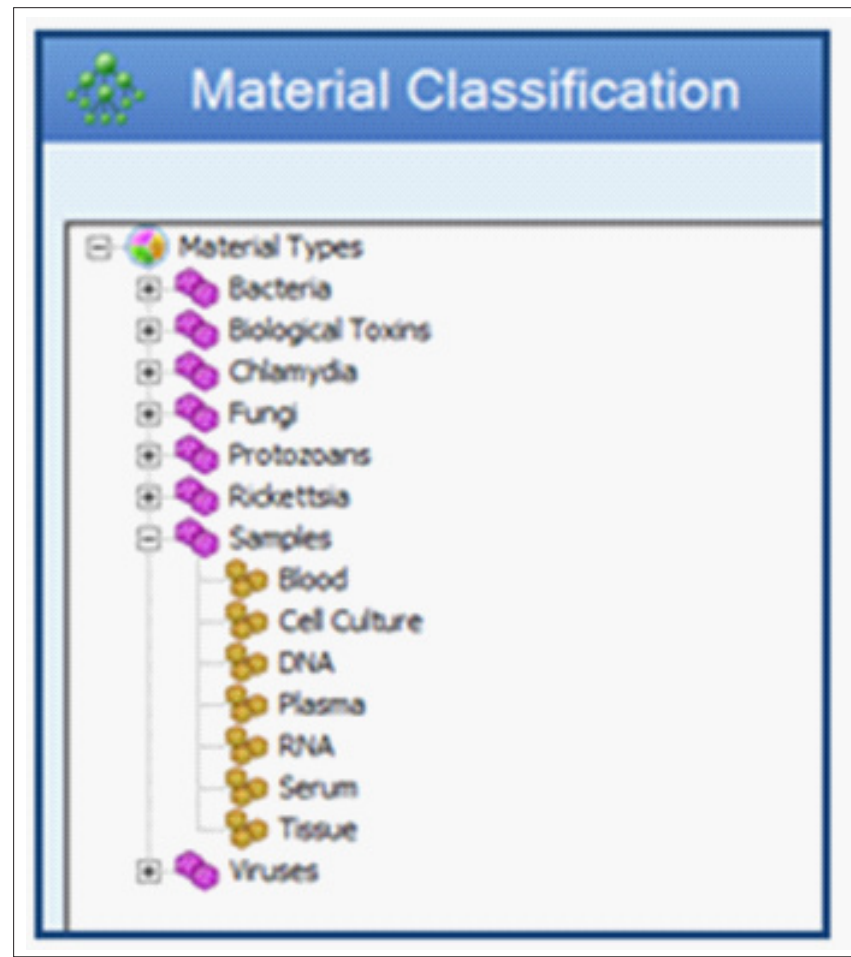

FIGURE 5: Material classification.

Electronic Integrated Disease Surveillance System provides means for integration with other local or international systems: the WHO module can transfer approved reports to
Computerised Information System for Infectious Diseases (CISID) assisting in International Health Regulations (IHR) compliance, open architecture approach supported by EIDSS allows to establish communications with existing local electronic systems. Regional cooperation between neighbouring countries is also possible on the base of EIDSS.

Full localisation of the system which includes electronic forms, reports and manuals, unified design and staff training make it easy to operate for non-experienced users. Turn-key deployment and support contribute to the sustainability of the solution.

\section{Results}

Electronic Integrated Disease Surveillance System is being developed since 2005 in collaboration with different institutions including the Centers for Disease Control and Prevention (CDC), US, the Walter Reed Army Institute for Research (WRAIR), US, Ministries of Health and Veterinary Departments of Kazakhstan, Uzbekistan, Azerbaijan and Georgia, Ukraine and Armenia and numerous international medical and veterinary experts. Following iterative approach EIDSS went through the number of expertise (more than 75000 hours) and revisions.

Currently version 3 is deployed in Azerbaijan (90 installations), Kazakhstan (more than 150 installations) and Georgia (more than 120 installations). The initial phase of deployment in Armenia and Ukraine. Electronic Integrated Disease Surveillance System has been officially recognised in Azerbaijan in 2010 and recently in Kazakhstan and Georgia.

Electronic Integrated Disease Surveillance System is a solution that improves the capacity to detect, diagnose and report bioterrorism attacks and potential pandemics, and supports bioresearch. It strengthens both regional and global disease surveillance with plans for expansion into African and Asian regions and globally.

\section{Method 2: Biological pathogens tracking using Pathogen Asset Control System}

Pathogen Asset Control System helps to track and control pathogens that are collected, investigated and stored in biological laboratories. It allows monitoring of the whole life-cycle of the pathogens with necessary levels of detail and appropriate security.

Pathogen Asset Control System can be implemented within different facilities, from small repositories with one centralised storage area to big multi-discipline institutions with distributed structure and numerous laboratories. Serverclient architecture allows immediate access to all necessary information for every user who has appropriate rights, which includes principal investigators, facility management, IT administrators and other laboratory personnel. 


\begin{tabular}{|c|c|c|c|c|c|}
\hline \multicolumn{6}{|c|}{ Freezer 1 [HSDO-1303].Shelf 1.Box 1} \\
\hline \multicolumn{2}{|c|}{ Container ID } & Pos & Material \# & Container Type & $\begin{array}{l}\text { Microorganism I } \\
\text { Sample type }\end{array}$ \\
\hline 1 & C100061 & 4 & M100008 & Ampoule & Brucella suis \\
\hline 2 & C100062 & 5 & M100008 & Ampoule & Brucella suis \\
\hline 3 & C100066 & 18 & M100008 & Ampoule & Brucella suis \\
\hline
\end{tabular}

FIGURE 6: Freezer content report.

Pathogen Asset Control System utilises Barcode technology and Radio-frequency Identification (RFID) to uniquely track all individual vials with highly pathogenic materials and allows error-free fast data input and retrieval.

Handling dangerous biological materials and processing information about them requires certain level of safety and security, and PACS provides invaluable assistance in this area. Separated access control to data, detailed audit trails, inventory audit function and other security features of the application help the owner to comply with international and local regulations in Biosafety and Biosecurity.

Considering that different organisations might have different requirements on what information must be tracked on biological materials and how, PACS allows several options for system customisation and adoption to local requirements. That includes designing barcode labels, managing repository configuration, creating different data entry forms depending on material type customise data entry forms and output reports. Being flexible and adoptable to the owner's requirements PACS employs all common operations with biological materials, such as registration, transfer, destruction, splitting and reculturing. All operations are supplied with printable reports and acts.

Pathogen Asset Control System provides wide opportunities to utilise information entered into the system for biological investigation support. A variety of different user defined fields and information in them, supplemented with the capability of creating custom reports provides an opportunity to output data in ways so it can be used for analysis of different parameters.

\section{Results}

Pathogen Asset Control System is an important part of the overall Biosecurity solution and can be very efficient in every biological laboratory where implemented. It allows the necessary levels of tracking and control over dangerous pathogens and complies with strengthening or enforcing regulations in Biosafety.

Being implemented in numerous laboratories in 6 different countries PACS proved its reliability and effectiveness. Pathogen Asset Control System is currently undergoing Certification and Accreditation (C\&A) process according to United States Department of Defense standards and should be offered to many government agencies as a system for tracking biological agents once C\&A is completed. Pathogen Asset Control System is a robust electronic system that competently helps to solve the important task of managing dangerous materials and will be in demand by biological laboratories.

\section{Acknowledgements}

Mr Tom G. Wahl is a project manager for EIDSS and PACS product. He prepared the overview of the EIDSS and PACS and introduction. Dr Aleksey V. Burdakov made contribution to all sections of the article, including problem statement. $\mathrm{Mr}$ Andrey O. Oukharov wrote EIDSS section of the article. $\mathrm{Mr}$ Azamat K. Zhilokov wrote PACS section.

\section{References}

Burdakov, A.V., 2005, 'Implementation of the communications and information technology part of the International Program on Treat Agent Detection and Response in the Republic of Kazakhstan', Environment and Population Health 1, 37.

Wahl, T. \& Burdakov, A.V., 2007, 'Electronic Integrated Disease Surveillance System (EIDSS)', Advances in Disease Surveillance 2, 171. 